\title{
Expanding undergraduate medical education in British Columbia: a distributed campus model
}

A full description of the first stages of the expansion of undergraduate medical education is available at www.cmaj.ca/cgi/content/full/173/6/589/DC1

A $\mathrm{s}$ in the rest of Canada, British Columbia has a shortage of physicians in rural areas, particularly in the northern parts of the province and on the remoter parts of Vancouver Island. People living in these areas have reduced access to health care and poorer health outcomes compared with people in the rest of BC. ${ }^{1}$ In Canada, more than 9 million people, $30.4 \%$ of the population, live in predominantly rural regions. $\mathrm{Al}$ though $20.6 \%$ of Canadian residents live in towns of under 10000 people, they are served by only $9.3 \%$ of the country's physicians.

The province has 1 accredited medical school, at the University of British Columbia (UBC). In 2003 its intake of students was 128 , despite a projected annual need in BC of at least 300 physicians. $^{2}$

This situation led in 2000 to the development of an academic, community and political will to address the growing physician shortage quickly, by means that would fit the geography and culture of BC. Citizen protests in rural areas and a strike in 2000 by physicians in the northern city of Prince George further focused attention on the distribution of health resources.

There are 2 broad educational approaches to increasing the number of physicians per capita and correcting the rural-urban maldistribution: the distributed approach, in which the medical school that is already established develops branch operations in remote areas, and new start, which involves building an additional medical school in a remote area. Both have been tried.

There is growing evidence that students from smaller com-

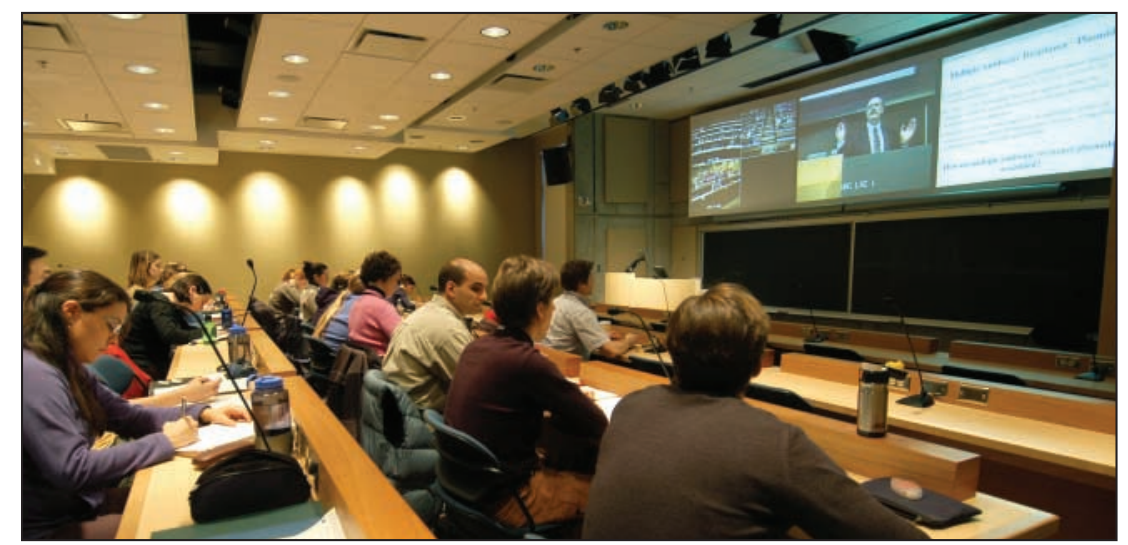

munities are more likely to end up working in rural areas than those from larger ones, and that physicians are more likely to work near where they trained. ${ }^{3}$ On that basis, UBC created its own solution to BC's shortage of northern and rural physicians by developing 3 geographically distributed campuses in partnership with 2 existing universities: the University of Victoria, in Victoria on Vancouver Island, and the University of Northern British Columbia (UNBC) in Prince George, an inland community about $1000 \mathrm{~km}$ north of Vancouver. The goal was to rapidly double the number of students admitted to medicine annually and to permit a portion of entering students to complete almost all of their undergraduate education at a single site of their choice.

All students are UBC students, who graduate with UBC degrees. Students spend their first semester at the UBC campus in Vancouver, and then go to their respective distributed campus for the next $3 \frac{1}{2}$ years. An important part of this solution was ensuring that all students have had access to all UBC student-support services regardless of their geographic site of instruction. Students at distributed campuses also have full access to the supports and services available locally.

UBC curriculum is problembased: from their first year, students study a case per week in 3 weekly small-group sessions. This is supplemented by lectures, laboratories, clinical skills sessions in local hospitals and visits to the offices of family physicians. As this accredited program was to be followed at the same schedule at all 3 campuses, basic science facilities including anatomy laboratories were required at all sites. Government financial support in 2002 led to new buildings on all 3 sites by 2004, recruitment of academic and administrative staff, and the adoption of a model for distributing the curriculum assisted by audiovisual technology. To deliver the new curriculum the 2 new distributed sites recruited basic scientists, academic physicians and new clinical faculty over an 18-month period, building on existing clinical faculty who support established residency programs. Comprehensive faculty development programs were put in place.

The admissions process was altered to involve clinical and academic faculty as well as the lay community from across the 


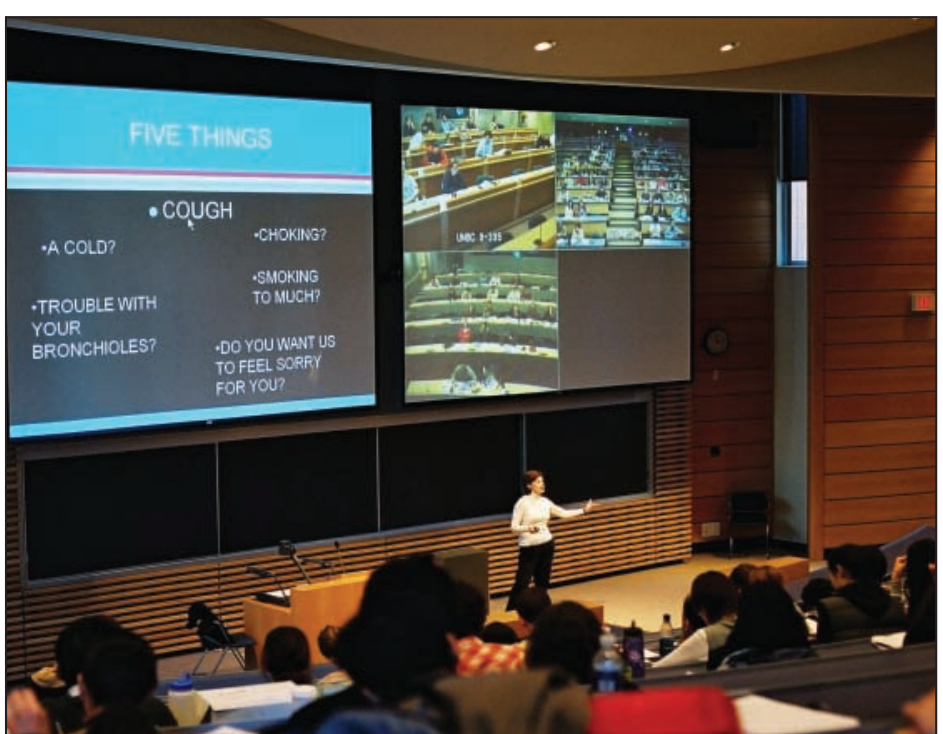

province; every student has been interviewed by a panel of 3 representatives from the respective areas: the established campus, Vancouver Island and northern and rural areas. UBC and UNBC developed a "rural suitability instrument" to select students for the distributed campuses; this tool is still being evaluated. Admitted students are being placed at their preferred site whenever possible. Applicants rose in number from 929 in 2003 to 1314 a year later, and continue to rise.

Bridging the geographic distances has been a vital element of this project. Advanced technology was developed to connect all 3 sites, based on the existing BCNet network that links the universities in the province. Because the lecture theatres and histology and anatomy laboratories at all campuses are interconnected, faculty at any site can deliver a lecture to the collaboration, enriching the curriculum for all participants. These facilities are fully interactive, with all instructors and students able to see and hear each other. The experience so far has been very positive, with excellent image quality and negligible time lag between sound and image. For- mal evaluation has suggested that the learning experience from a distributed lecture is as good as that from a live lecture.

The availability of videoconferencing rooms also facilitates course and curriculum committee and faculty support meetings. The technology has proven to be reliable, with minimal failures during the first 3 months of operation; we credit this to meticulous planning and rigorous testing.

The first students in this distributed, expanded program have now completed their first year. We are still learning how to manage a distributed model and discovering unexpected problems; nonetheless, the positive feedback from students and faculty is testimony to the detailed project planning, piloting and professional management that preceded implementation. Inter-university collaboration along with a sense of achievement at university, government and community levels at our innovative management of such a rapid growth in undergraduate numbers. Developing administrative systems across 3 different universities has been a major challenge, as has the develophas strengthened and grown, ment of new cultures of medical education and student support in the distributed campuses.

A comprehensive evaluation has been implemented, but it will be a decade before we know what effect we have made on the numbers of Canadiantrained physicians in the small communities, rural and northern areas of BC. The first signs, however, are encouraging.

\section{David Snadden}

Associate Dean

Northern Medical Program

Associate Vice President, Medicine

University of Northern British

Columbia

Prince George, BC

Joanna Bates

Senior Associate Dean, Education

University of British Columbia

Vancouver, BC

on behalf of the UBC Associate

Deans of MD Undergraduate

Education

Associate Deans of MD Undergraduate Education, University of British Columbia: Joanna Bates, Senior Associate Dean, Education, University of British Columbia; Oscar Casiro, Associate Dean, Island Medical Program, and Head, Division of Medical Sciences, University of Victoria; Bruce Fleming, Associate Dean, Student Affairs, and Vera Frinton, Associate Dean, Admissions, University of Northern British Columbia; and David Snadden, Associate Dean, Northern Medical Program, and Associate Vice President, Medicine, University of Northern British Columbia; and Angela Towle, Associate Dean, MD Undergraduate Curriculum and Vancouver Fraser Medical Program, University of British Columbia.

\section{References}

1. Provincial Health Officer. The bealth and well-being of people in British Columbia: Provincial Health Officer's annual report 2002. Victoria: The Office; 2002. Available: www.healthservices.gov .bc.ca/pho/ar (accessed 2005 Aug 06).

2. British Columbia Medical Association. Physician supply and distribution in $B C$ - fact sheet. Vancouver: The Association; 2005. Available: www.bcma .org/public/negotiations information /physiciansfactsheet.htm (accessed 2005 Jul 21).

3. Rourke J, for the Task Force of the Society of Rural Physicians of Canada. Strategies to increase the enrolment of students of rural origin in medical school: recommendations from the Society of Rural Physicians of Canada. CMA7 2005;172(1):62-5. 